\title{
A genome-wide expression analysis identifies a network of EpCAM-induced cell cycle regulators
}

\author{
K Maaser' and J Borlak*,I \\ 'Molecular Medicine and Medical Biotechnology, Fraunhofer Institute of Toxicology and Experimental Medicine, Nikolai-Fuchs-Str. I, 30625 Hannover, \\ Germany
}

Expression of the epithelial cell adhesion molecule EpCAM is upregulated in a variety of carcinomas. This antigen is therefore explored in tumour diagnosis, and clinical trials have been initiated to examine EpCAM-based therapies. Notably, the possible intracellular effects and signalling pathways triggered by EpCAM-specific antibodies are unknown. Here, we show treatment of the mouse lung carcinoma cell line $\mathrm{A} 2 \mathrm{Cl}$, of the human lung carcinoma cell line A549 and the human colorectal cell line Caco-2 with the monoclonal EpCAM antibody G8.8 to cause dose dependently an increase in cell proliferation, as determined by the MTS and the 5'-bromo-2'-deoxyuridine (BrdU) labelling assay. Furthermore, a genome-wide approach identified networks of regulated genes, most notably cell cycle regulators, upon treatment with an EpCAM-specific antibody. Indeed, changes in the expression of cell cycle regulators agreed well with the BrdU labelling data, and an analysis of differentially expressed genes revealed the processes with the strongest over-representation of modulated genes, for example, cell cycle, cell death, cellular growth and proliferation, and cancer. These data suggest that EpCAM is involved in signal transduction triggering several intracellular signalling pathways. Knowing EpCAM signalling pathways might lead to a reassessment of EpCAM-based therapies.

British Journal of Cancer (2008) 99, 1635-1643. doi:I0.1038/sj.bjc.6604725 www.bjcancer.com

Published online 28 October 2008

(c) 2008 Cancer Research UK

Keywords: epithelial cell adhesion molecule (EpCAM); lung carcinoma; proliferation; DNA microarray analysis; proliferation; cell cycle

The epithelial cell adhesion molecule (EpCAM) was initially described as tumour-associated antigen (Koprowski et al, 1979). However, EpCAM was then shown to be a panepithelial marker that is enriched at the basolateral membrane (Winter et al, 2003b). The level of EpCAM expression has been associated with cellular differentiation, for example the germinal regions in normal colonic crypts display high levels of EpCAM expression, which decrease as cells differentiate and migrate to the top of the villi (Schiechl and Dohr, 1987).

Expression of EPCAM is known to be upregulated in a variety of carcinomas including those of the lung and colon (Went et al, 2006). Recent evidence is suggestive of EpCAM to serve as a cancer stem cell marker (Yamashita et al, 2007). The correlation between EpCAM expression and prognosis seems to be tissue specific. In breast, ovarian, and oesophageal squamous cell carcinoma, EpCAM overexpression has been correlated with poor prognosis (Spizzo et al, 2002, 2004, 2006; Stoecklein et al, 2006). In contrast, a positive impact of EpCAM overexpression on survival was found in renal cell carcinoma and the subgroup of pT2 adenocarcinomas of the lung (Seligson et al, 2004; Songun et al, 2005). On account of its high level of expression in several carcinomas, it is a candidate target for tumour diagnosis and therapy. Several clinical trials targeting EpCAM have been conducted with variable success (Baeuerle and Gires, 2007). Mouse-derived monoclonal antibodies

\footnotetext{
*Correspondence: Professor Dr J Borlak;

E-mail: borlak@item.fraunhofer.de

Received I 5 September 2008; accepted 16 September 2008; published online 28 October 2008
}

directed to EpCAM have been successfully used for adjuvant treatment of minimal residual disease of colon carcinoma (Riethmuller et al, 1994, 1998). Initially, the approach appeared to improve long-term survival of patients, but a larger study could not corroborate these promising therapeutic effects (Punt et al, 2002). At present, there are several ongoing clinical trials using murine or human antibodies directed against EpCAM (reviewed in Armstrong and Eck, 2003; Baeuerle and Gires, 2007). In vitro studies demonstrated several possible mechanisms of action. Antitumoral effects have been ascribed to antibody- and complement-dependent cellular toxicity or anti-ideotypic immune response (Fagerberg et al, 1995; Armstrong and Eck, 2003). Therefore, targeting EpCAM for immunotherapy mainly aims to break self-tolerance towards EpCAM. However, recent studies showed that EpCAM itself can function as a signalling molecule.

Epithelial cell adhesion molecule is a membrane protein with two EGF-like repeats, followed by a cysteine-poor region and a short cytoplasmic tail (Baeuerle and Gires, 2007). It is known as a $\mathrm{Ca}^{2+}$-independent, homophilic cell-cell adhesion molecule. However, there is growing evidence that EpCAM plays a functional role not only in cell adhesion, but also in diverse processes such as signalling, cell migration, differentiation, and proliferation. Despite its function as a cell adhesion molecule, it has been associated with metastasis formation (Gastl et al, 2000; Spizzo et al, 2004; Varga et al, 2004), and silencing of EpCAM expression was shown to decrease the migration rate (Osta et al, 2004; Winter et al, 2007). Epithelial cell adhesion molecule has been shown to be involved in the abrogation of E-cadherin-mediated cell-cell interactions by disrupting the link between $\alpha$-catenin and F-actin 
(Winter et al, 2003a). Epithelial cell adhesion molecule itself has been shown to interact through $\alpha$-catenin with the actin cytoskeleton, thereby inducing the formation of stress fibres (Guillemot et al, 2001). Moreover, a direct impact of EpCAM on cell cycle control by upregulating the proto-oncogene c-myc and the cyclins A and E has been reported (Munz et al, 2004). Recently, it has been shown that EpCAM formed complexes with the tightjunction protein claudin-7, the variant isoform of the cell-matrix adhesion protein $\mathrm{CD} 44 \mathrm{v} 6$, and the tetraspanin CD9, which facilitated metastasis formation (Kuhn et al, 2007). Thus, EpCAM signalling might depend on the microenvironment and interaction with other membrane molecules as well as on the expression level and subsequent possible oligomerisation. However, the exact mechanisms of EpCAM signalling are yet to be elucidated.

In this study, we investigated the EpCAM-mediated signalling upon crosslinking with a monoclonal rat anti-human antibody G8.8. Therefore, we used a novel mouse lung carcinoma cell line that was isolated from the lung tumour of a c-raf/c-myc doubletransgenic mouse as a model to investigate EpCAM signalling. Moreover, we compared the results obtained from this cell model with the two human cell lines A549 (lung carcinoma) and Caco-2 (colorectal carcinoma), both of which are well known to express EpCAM highly, with the goal of understanding EpCAM signalling across a broader scope of different tumour origins.

\section{MATERIALS AND METHODS}

\section{Cell culture}

The spontaneously transformed mouse lung carcinoma cell line A2C12 was isolated from lung tumours of double c-myc and c-raf transgenic mice. Cells were isolated from lung tumours of 8-month-old transgenic mice using the protocol for the isolation and culture of rodent primary respiratory cells (Hansen et al, 2006). The human colorectal adenocarcinoma cell line Caco-2, the human lung carcinoma cell line A549, and the mouse cell line A2C12 were cultured in Dulbecco's modified Eagle's medium supplemented with $10 \%$ foetal calf serum, $2 \mathrm{~mm}$ L-glutamine, $100 \mathrm{U} \mathrm{ml}^{-1}$ streptomycin, and $100 \mu \mathrm{g} \mathrm{ml}^{-1}$ penicillin in a humidified atmosphere containing $5 \% \mathrm{CO}_{2}$ at $37^{\circ} \mathrm{C}$.

\section{Western blot analyses}

Whole-cell extracts were prepared by harvesting and lysing the cells with lysis buffer $\left(50 \mathrm{~mm}\right.$ Tris $(\mathrm{pH} 6.8), 1.5 \% \mathrm{w} \mathrm{v}^{-1}$ sodium dodecyl sulphate). Samples were boiled for $10 \mathrm{~min}$, sonicated 20 times at an interval setting of 0.5 (UP 200 s; Dr Hielscher, Teltow, Germany) in $500 \mu \mathrm{l}$ lysis buffer on ice, and centrifuged at $12000 \mathrm{~g}$ for $10 \mathrm{~min}$ at room temperature. The supernatant was recovered. Protein content of the lysate was determined by Smith protein assay.

Eighty micrograms of total protein extracts were separated on a $12 \%$ SDS - polyacrylamide gel and blotted onto a PVDF membrane in $25 \mathrm{~mm}$ Tris and $190 \mathrm{~mm}$ glycin at $4^{\circ} \mathrm{C}$ for $2 \mathrm{~h}$ at $350 \mathrm{~mA}$. Blots were blocked in Rotiblock (Roth, Karlsruhe, Germany) for $1 \mathrm{~h}$ and then incubated overnight at $4{ }^{\circ} \mathrm{C}$ with rabbit anti-human antibodies Raf-1 (C-12), c-Myc (N-262), CD44 (N-18), or CD133 (K-18) (all of which are also recommended to detect mouse antigens respectively; $1 \mu \mathrm{g} \mathrm{ml}^{-1}$; Santa Cruz, Heidelberg, Germany), or rat antimouse EpCAM antibody $\left(2 \mu \mathrm{g} \mathrm{ml}^{-1}\right.$; kindly provided by Micromed, Munich, Germany). Results for CD133 expression in mouse A2C12 were confirmed using rat anti-mouse CD133 antibody (13A4, $2.5 \mu \mathrm{g} \mathrm{ml}^{-1}$; ebioscience, San Diego, CA, USA), with protein lysate of mouse kidney as a positive control. After washing with Trisbuffered saline ( $25 \mathrm{~mm}$ Tris and $135 \mathrm{~mm} \mathrm{NaCl}$; pH 7.6) containing $0.1 \%$ Tween and incubation with horseradish peroxidase-coupled anti-IgG antibody (1:10000; Chemicon, Temecula, CA, USA) at room temperature for $1 \mathrm{~h}$, the blot was washed extensively, developed using enhanced chemiluminescent detection (PerkinElmer, Jügesheim, Germany), and recorded with Kodak IS $440 \mathrm{CF}$ (Kodak; Biostep GmbH, Jahnsdorf, Germany).

\section{Flow cytometry}

Cells were trypsinised, washed twice with phosphate-buffered saline (PBS; $140 \mathrm{~mm} \mathrm{NaCl}, 10 \mathrm{~mm} \mathrm{Na} 2 \mathrm{HPO}_{4}, 2.6 \mathrm{~mm} \mathrm{KCl}, 1.4 \mathrm{~mm}$ $\mathrm{KH}_{2} \mathrm{PO}_{4}$ ( $\left.\mathrm{pH} 7.4\right)$ ), and immunoassayed, as described previously (Maaser et al, 2001). Cells were incubated for $1 \mathrm{~h}$ at room temperature with primary rabbit anti-mouse CD44 or rabbit antihuman CD133 (both $4 \mu \mathrm{g} \mathrm{ml}^{-1}$; Santa Cruz), or with rat anti-mouse EpCAM antibody $\left(10 \mu \mathrm{g} \mathrm{ml}^{-1}\right.$; kindly provided by Micromed), respectively. Results of EpCAM expression in human cell lines were confirmed using mouse anti-human EpCAM antibody $\left(10 \mu \mathrm{g} \mathrm{ml}^{-1}\right.$, clone 0.N.276; Santa Cruz). Cells were washed twice with PBS and then incubated with $4 \mu \mathrm{g} \mathrm{ml}^{-1}$ secondary Alexa ${ }^{\mathrm{TM}}$ 488-labelled goat anti-rabbit (CD44 and CD133) or goat anti-rat (EpCAM) IgG antibody (Molecular Probes, Eugene, OR, USA), respectively, for $1 \mathrm{~h}$ at room temperature. Donkey anti-mouse IgG ( $4 \mu \mathrm{g} \mathrm{ml}^{-1}$; Dianova, Hamburg, Germany) was used as secondary antibody for the mouse anti-human EpCAM antibody. Fluorescence was detected by flow cytometry on a FACSscan (Becton Dickinson, Heidelberg, Germany) and analysed using CellQuest software.

\section{Cell proliferation}

Cells were plated in 96-well microtitre plates at a density of 5000 cells per well $24 \mathrm{~h}$ before treatment. Cells were treated with monoclonal rat anti-mouse EpCAM antibody at concentrations of $1-100 \mu \mathrm{g} \mathrm{ml}^{-1}$ for $48 \mathrm{~h}$. Cell proliferation was measured using two different methods: incorporation of $5^{\prime}$-bromo- $2^{\prime}$-deoxyuridine (BrdU) and metabolic conversion of 3-(4,5-dimethylthiazol-2-yl)5-(3-carboxymethoxyphenyl)-2-(4-sulphophenyl)-2H-tetrazolium (MTS).

Incorporation of $\mathrm{BrdU} \quad 5^{\prime}$-Bromo- $2^{\prime}$-deoxyuridine incorporation was measured using BrdU Cell Proliferation Assay (Merck, Darmstadt, Germany) according to the manufacturer's instructions. Cells were labelled with $\operatorname{BrdU}(1: 100)$ for the last $4 \mathrm{~h}$ of incubation. Cells were washed, fixated, and incubated with mouse anti-BrdU antibody $(1: 100 ; 100 \mu \mathrm{l}$ per well) for $1 \mathrm{~h}$ at room temperature. Antibody labelling was detected by secondary peroxidase-coupled goat anti-mouse antibody $(1: 1000 ; 100 \mu \mathrm{l}$ per well; $30 \mathrm{~min}$ at room temperature). After washing, peroxidase substrate was added for $15 \mathrm{~min}$. The peroxidase reaction was stopped by adding $100 \mathrm{ml} 2.5 \mathrm{~N}$ sulphuric acid, and absorbance was measured using dual wavelengths 450 and $595 \mathrm{~nm}$.

MTS assay The CellTiter $96^{\mathbb{R}} \mathrm{AQ}_{\text {ueous }}$ Non-Radioactive Cell Proliferation Assay (Promega, Mannheim, Germany) was used to determine the number of viable cells in culture. The MTS assay is based on the ability of viable cells to convert a soluble tetrazolium salt to a formazan product. After exposure to rat anti-mouse EpCAM antibody, MTS reagent was added and cell cultures were incubated at $37^{\circ} \mathrm{C}$ for $1 \mathrm{~h}$. Absorbance was recorded at $492 \mathrm{~nm}$ (Victor multireader; PerkinElmer).

\section{Isolation of RNA, production of c-RNA, array hybridisation, and scanning}

The c-RNA samples were prepared following the Affymetrix Gene Chip ${ }^{\circledR}$ Expression Analysis Technical Manual (Santa Clara, CA, USA). Ten micrograms of biotinylated fragmented cRNA were hybridised onto the Mouse Genome 430A 2.0 Array or the Human Genome U133 Plus 2.0 Array, respectively. The procedures for 
isolation of RNA, production of c-RNA, array hybridisation, and scanning were performed according to Affymetrix's manual and as described earlier (Borlak et al, 2005). Each hybridisation image was scaled to probe set intensity of 250 for Mouse Genome 430A 2.0 Arrays and of 200 for the Human Genome U133 Plus 2.0 Arrays for comparison between chips.

\section{Gene expression data analysis}

Data analysis was performed as described earlier (Borlak et al, 2005). For single arrays, a statistical expression algorithm within the Affymetrix ${ }^{\circledR}$ Microarray Suite software (version 5) yielded gene expression values as numeric values (signal intensities) and detection calls ('present' or 'absent') produced with different algorithms. A comparison between two arrays (tumour and control lung) resulted in the signal logarithm ratio ( $\log 2$ ratio) and a change call ('increase' or 'decrease') for the expression level of each gene. The group of differentially expressed genes was restricted to those genes that were detected ('present' call) in antibody-treated samples for the upregulated genes and in all control samples for the downregulated genes. Further criteria were a minimum intensity of 100 in treated samples, a fold change $>2$, and $100 \%$ of 'increase' calls in comparative ranking analysis for treated $v s$ untreated samples for upregulated genes, and a minimum intensity of 100 in control samples, a fold change $<-2$, and $100 \%$ of 'decrease' calls accordingly for downregulated genes. The list of differentially expressed genes was submitted to Ingenuity Pathway Analysis 5.5 (www.ingenuity.com) to analyse gene functions. The significance of functional enrichment was computed by a Fisher's exact test and represented by a range of $P$ values associated with specific functions.

\section{Reverse transcription and real-time semiquantitative PCR}

Total RNA from each sample $(2 \mu \mathrm{g})$ was used for reverse transcription (Omniscript Reverse Transcriptase; Qiagen $\mathrm{GmbH}$, Hilden, Germany). Real-time PCR was performed in a mixture containing a c-DNA equivalent to $25 \mathrm{ng}$ of total RNA, $1 \mu \mathrm{m}$ of each primer, $0.5 \mathrm{~mm}$ dNTP mixture, 0.625 U Thermostart-Taq (ABgene, Hamburg, Germany), and $1 \times$ PCR buffer (ABgene), $0.66 \mathrm{mg} \mathrm{ml}^{-1}$ bovine serum albumin, $1.75 \mathrm{~mm} \mathrm{MgCl}_{2}$, and 5\% SYBR Green (Roche Diagnostics, Mannheim, Germany) in a total volume of $20 \mu \mathrm{l}$. Realtime RT - PCR measurement was performed with the LightCycler ${ }^{\mathrm{R}}$ (Roche Diagnostics, Mannheim, Germany). Experimental conditions and detailed oligonucleotide sequence information are given in Supplementary Table S1. Specificity of primers was confirmed by agarose-gel electrophoresis of PCR products. Differences in gene expression are shown as fold changes of antibody-treated probes $v s$ untreated probes and calculated by $\Delta \Delta C_{\mathrm{t}}$ values. Mean expression values of the mitochondrially encoded ATP synthase 6 (MT-ATP6) and glyceraldehyde-3-phosphate dehydrogenase (GAPDH) were taken as housekeeping genes.

\section{RESULTS}

\section{Expression of c-raf, c-myc, EpCAM, CD133, and CD44}

The cell line $\mathrm{A} 2 \mathrm{C} 12$ was isolated from lung carcinomas of mice that were double transgenic for c-myc and c-raf-1-BxB. Expression of c-myc, c-raf, and transgenic c-raf-1BxB was detected by immunoblot. The expression of wild-type c-raf $(72 \mathrm{kDa})$ in $\mathrm{A} 2 \mathrm{C} 12$ cells was markedly stronger than in non-neoplastic lung tissues, A549 cells, or Caco-2 cells (Figure 1A). However, the transgenic expression of c-raf- $1 \mathrm{BxB}(42 \mathrm{kDa})$ could not be detected in these cells, indicating that $\mathrm{A} 2 \mathrm{C} 12$ cells lost the transgenic c-raf- $1 \mathrm{BxB}$. Expression of c-myc was detectable in all cell lines as well as in non-neoplastic lung tissue.

All investigated cancer cell lines expressed EpCAM, as revealed by western blot analysis. In contrast, no EpCAM expression was found in non-cancerous lung tissue (Figure 1A). Expression of EpCAM in the cancer cell lines was confirmed by flow cytometry (Figure 1B).

Epithelial cell adhesion molecule was found to be highly elevated in pre-malignant hepatic tissues (Kim et al, 2004) and was discussed as a marker for tumour progenitor cells (Yamashita et al, 2007). Therefore, we investigated whether the lung and colorectal carcinoma cells used in this study express additional tumour stem cell markers such as CD44 and CD133, as we showed that they expressed EpCAM.

Expression of CD44 was shown for all investigated carcinoma cell lines, but not in healthy mouse lung tissue, as determined by western blot analysis (Figure 1C). CD44 expression was higher in the mouse lung carcinoma cell line $\mathrm{A} 2 \mathrm{C} 12$, which was isolated from c-myc and c-raf transgenic mice, than in the human lung carcinoma cell line A549 and the human colorectal carcinoma cell line Caco-2. In contrast, the tumour stem cell marker CD133 was detected in Caco-2 cells only. mRNA expression analysis for CD133 and CD44 by microarray analysis resembled the protein expression results. No CD133 mRNA expression (detection call 'absent') could be detected in the lung carcinoma cell lines $\mathrm{A} 2 \mathrm{C} 12$ and $\mathrm{A} 549$, whereas a highexpression signal intensity was seen in Caco-2 cells (Figure 1C). CD44 mRNA was detected in all the three cell lines. Expression of CD44 protein was confirmed by flow cytometry (Figure 1D).

\section{Monoclonal anti-EpCAM antibody G8.8-induced proliferation}

After $48 \mathrm{~h}$ of treatment, monoclonal rat anti-mouse EpCAM antibody G8.8 dose-dependently increased the number of metabolically active cells. A significant increase in cell number was observed in the mouse lung carcinoma cell line A2C12 already at $10 \mu \mathrm{M}$ antibody treatment and in the human colorectal carcinoma cell line Caco-2 at $100 \mu \mathrm{M}$ antibody treatment (Figure 2A). In the human lung carcinoma cell line A549, anti-EpCAM antibody induced a slight (at $10 \mu \mathrm{m}$ up to $114 \%$ as compared with untreated control), but not significant, increase (data not shown).

Next, BrdU incorporation was measured to assess the proliferation-modulating effects of the anti-EpCAM antibody G8.8. In A2C12 cells as well as in A549 cells, a significant increase in BrdU incorporation was detected upon treatment with $100 \mu \mathrm{M}$ antiEpCAM for $48 \mathrm{~h}$ (Figure 2B). However, in contrast to the proliferation-inducing effects measured by MTS assay, no changes in BrdU incorporation could be measured in Caco- 2 cells. This might be due to the long generation time of about $62 \mathrm{~h}$ of Caco-2 cells, leading to a lower sensitivity of the BrdU assay in these cells.

\section{Anti-EpCAM antibody-induced modulation of gene expression}

The genome-wide expression profile of EpCAM antibody-treated cells was analysed to investigate the molecules involved in EpCAM signalling. Epithelial cell adhesion molecule antibody modulated gene expression in all investigated cell lines, however, to different extents (Supplementary Table S2). Gene expression modulation was most prominent in mouse and human lung carcinoma cell lines, A2C12 and A549, in both of which expression of more genes was downregulated than upregulated. In A2C12 cells, anti-EpCAM antibody treatment modulated the expression of 1973 gene transcripts (probe sets), $901(46 \%)$ of which were increased and $1072(54 \%)$ decreased. The modulation of gene transcription was similarly high in A549 cells (1743 modulated gene transcripts, 598 (34\%) of which were increased and 1154 (66\%) decreased). In the colon adenocarcinoma cell line Caco-2, less genes were modulated in total, with a higher extent of upregulation than downregulation. In Caco-2 cells, the expression of 378 gene transcripts was changed, $246(65 \%)$ of which were increased and $142(35 \%)$ decreased. 
Analysis of function of differentially expressed genes was computed using Ingenuity Pathway Analysis software. Interestingly, the processes with the strongest over-representation of differentially expressed genes were the same for A1C12, A549, and Caco-2 cells.
These processes include cell death, cell signalling, cellular growth and proliferation, cancer, cell cycle, and gene expression (Table 1A).

We further analysed genes related to cell cycle modulation with respect to their relevance for the distinct phases of the cell cycle.
A

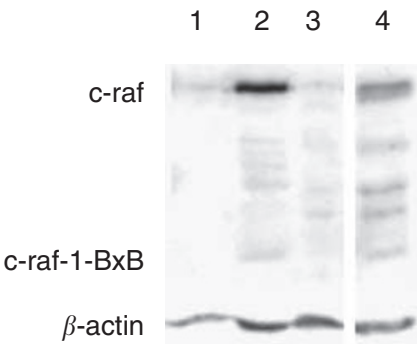

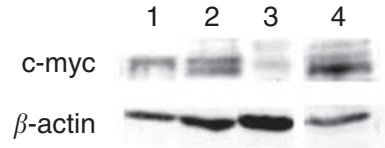

EpCAM

$\beta$-actin

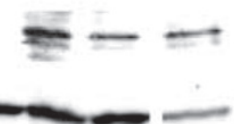

B
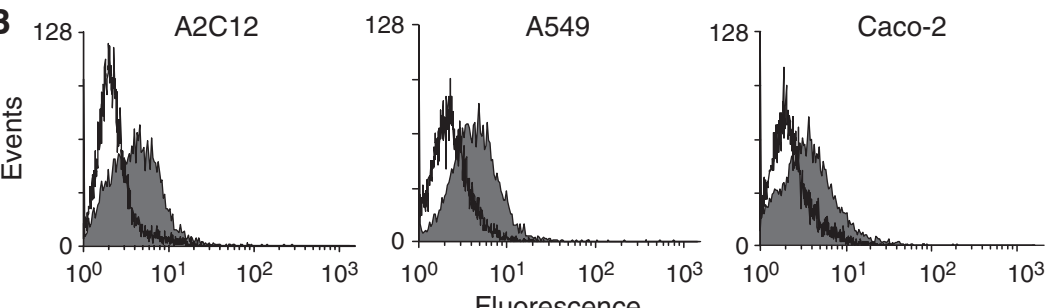

Fluorescence
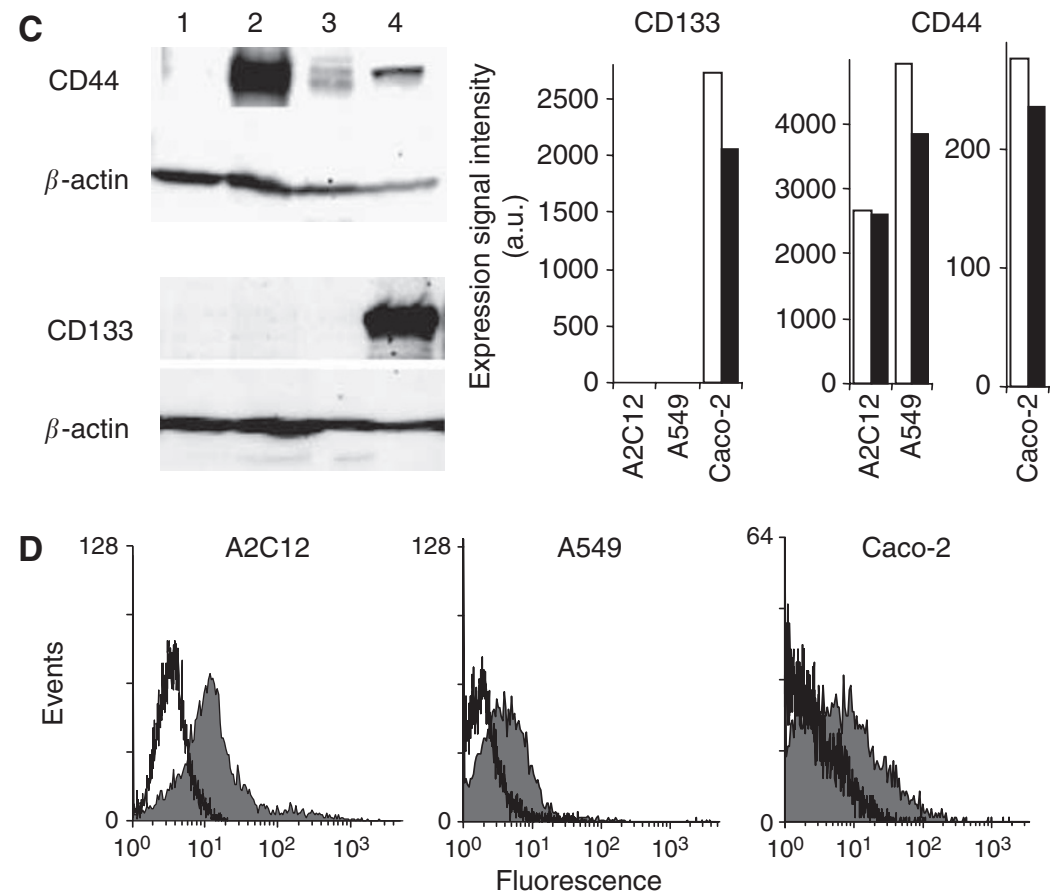

Figure I Expression of c-raf, c-myc, EpCAM, CD44, and CDI33. (A) Expression of c-raf in A2CI2 cells (lane 2) was markedly stronger than that in nonneoplastic lung tissue (lane I), A549 cells (lane 3), or Caco-2 cells (lane 4). Expression of c-myc was detected in non-neoplastic lung tissue (lane I) as well as in A2CI 2 cells (lane 2), A549 cells (lane 3), and Caco-2 cells (lane 4). Epithelial cell adhesion molecule was found to be expressed in the cancer cell lines A2CI2 (lane 2), A549 (lane 3), and Caco-2 (lane 4), but not in non-cancerous lung tissue (lane I) as determined by western blot analysis. (B) Epithelial cell adhesion molecule expression in A2C12, A549, and Caco-2 cells was determined by flow cytometry using anti-EpCAM antibody G8.8. The mean fluorescence of EpCAM-labelled cells (filled curve) was increased 1.98-fold for A2CI2 cells, 1.92-fold for A549 cells, and I.46-fold for Caco-2 cells as compared with the respective controls (black line). (C) CD44 was found to be highly expressed in the mouse lung carcinoma cell line A2CI2 (lane 2), but not in non-cancerous lung tissue (lane I), as determined by western blot analysis. A low CD44 expression was found in the cell lines A549 (lane 3) and Caco-2 (lane 4). CD I33 was found to be expressed in Caco-2 cells (lane 4) only, but not in non-cancerous lung tissue (lane I), AICI2 cells (lane 2), and A549 cells (lane 3). Expression signal intensities for CDI33 and CD44 of control cells (white columns) and G8.8 antibody-treated cells (black columns), as determined by microarray analysis. In the case of more than one probe set per gene (CD44), highest intensities of the probe sets, which are specific for only one transcript designated as number at, are shown. Signal intensities of probe sets 1419700 a at (mouse CDI33), 204304 s at (human CDI33), I423760 at (mouse CD44), and 2I 2063_at (human CD44) are shown. (D) CD44 expression was confirmed for A2CI2, A549, and Caco-2 cells by flow cytometry. Cells were stained with the rat anti-human CD44 antibody (filled curve) or isotypic control antibody (black line) and secondary FITC-labelled anti-rat antibody. Results of one representative experiment each are shown. EpCAM = epithelial cell adhesion molecule. 

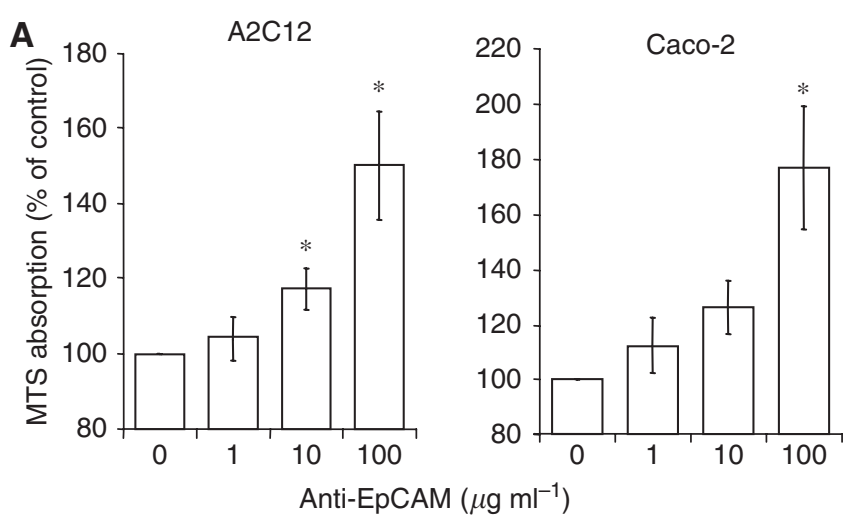

the G1/G0 phase are under-represented upon EpCAM antibody treatment, thereby reflecting the pro-proliferative effects of this treatment. Downregulated genes include CD44, met proto-oncogene (MET), and the apartyl-t-RNA synthetase (DARS). DARS is known to decrease G1/S-phase transition (Yamashita et al, 1999), and its downregulation by EpCAM antibody treatment might therefore contribute to the proliferative effects of antibody treatment. However, two genes exclusively linked to the G1/G0 phase were found to be upregulated in A2C12 and A549 cells, the cysteine-rich angiogenic inducer 61 (CYR61) and the dualspecificity phosphatase 1 (DUSP1). Other cell cycle-associated genes upregulated in $\mathrm{A} 2 \mathrm{C} 12$ and $\mathrm{A} 549$ cells include the superoxide dismutase 2 (SOD2, G1/G0 and S phases), basic helix-loop-helix domain containing class B 2 (BHLHB2, S phase), jun B protooncogene (JUNB, S and G2/M phases), jun oncogene (JUN, G1/G0 and G2/M phases), leukaemia inhibitory factor (LIF, G1/G0 and G2/M phases), and MDM2 (all cell cycle phases).

Three genes, which can be associated with specific cell cycle phases, were found to be commonly modulated in both human carcinoma cell lines A549 and Caco-2. The DNA-damage-inducible transcript 3 (DDIT3), also known as GADD153, and the growth differentiation factor 15 (GDF15), which have been associated with the G1 phase of the cell cycle, were found to be upregulated. The expression of thymidylate synthetase (TYMS, G1 and S phases) was downregulated in $\mathrm{A} 2 \mathrm{C} 12$ and $\mathrm{A} 549$ cells by EpCAM antibody treatment.

Most differentially expressed genes associated with cell cycle were related to specific cell cycle phases in A2C12 cells. Therefore, we focused on $\mathrm{A} 2 \mathrm{C} 12$ cells during single-gene analysis (Figure $3 \mathrm{~A}$ ). We identified mainly three functional groups of genes associated with cell cycle regulation: first, genes whose products are directly involved in cell cycle regulation such as cyclins, cyclin-dependent kinases, phosphatases, and cell cycle inhibitors; second, genes that code for growth factors, growth factor receptors, and their adaptor and signalling proteins. Moreover, several transcription factors were found to be differentially expressed. Several of these genes can be considered as p53 network (Figure 3B), which might play a role in the signal transduction leading to the proliferative effects of EpCAM antibody treatment. Epithelial cell adhesion molecule antibody altered proliferation similarly in the three investigated cell lines. We therefore focused on genes that were significantly modulated in A2C12, A549, and Caco-2 cells in parallel to further investigation of EpCAM signalling (Table 2). Interestingly, five out of 13 commonly modulated genes were related to cell cycleregulating or apoptotic functions. Expression of the genes positively regulating the cell cycle (LATS2, FOSL2, and PIM1) or inhibiting apoptosis (GADD45 and PIM1) was increased, whereas the expression of a pro-apoptotic gene transcript was decreased (DIDO1). Other genes differently regulated by anti-EpCAM antibody treatment include genes for three enzymes of biosyntheses (FDFT1, LCMT2, and MAT2A), one topoisomerase (TOP2A), one gene associated with protein folding (DNAJA1), and four transcripts of unknown functions (Table 2).

\section{Validation of microarray data by real-time PCR}

Expression of selected genes was investigated with real-time PCR using the LightCycler. Comparisons of fold changes determined by microarray analysis and real-time PCR are shown in Table 3 and Supplementary Figure S1. In almost all cases, the significant change found by microarray analysis could be confirmed by realtime PCR analysis. Although the level of altered expression varied, there was strong concordance between both methods. Only a few divergent results between the two methods have been detected, which might be due to the different probe sets/fragments of the specific gene targeted by the respective method. For example, a decreased expression of Trp53 in microarray analysis was measured only in two probe sets that recognise multiple alternative 
Table I Analysis of function of differentially expressed genes

Number of differentially expressed genes (induced/repressed)

\section{(A) Functional category}

Cell cycle ${ }^{b}$

Cellular growth and proliferation ${ }^{c}$

Cell death ${ }^{\mathrm{d}}$

Cancer $^{\mathrm{e}}$

\section{A2CI 2}

$232(126 / 106)$

$415(204 / 211)$

$395(202 / 193)$

$462(242 / 220)$

\section{A549}

$153(73 / 80)$

$306(139 / 167)$

$265(128 / 137)$

$344(155 / 189)$

\section{Caco-2}

$33(25 / 8)$

$46(33 / 13)$

$62(48 / 14)$

$77(54 / 23)$
A2CI2/A549

$29(20 / 9)$

$48(29 / 19)$

$49(33 / 16)$

$54(31 / 23)$

Number of differentially expressed genes (induced/repressed)

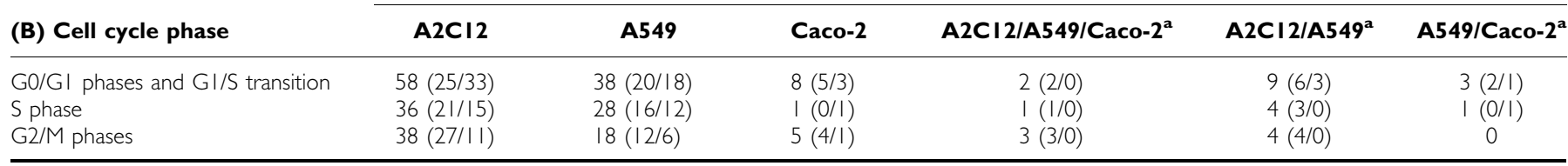

(A) Most significantly over-represented functional categories in the group of significantly regulated genes are shown. (B) Analysis of cell cycle-related differentially expressed genes. ${ }^{a}$ Commonly modulated in the cell lines mentioned. ${ }^{b}$ Includes the functions and stages of the cell cycle including cell division. Functions associated with mitosis and meiosis are included in this category. Some examples of functions in this category are assembly of telomeres, cell cycle progression, and G0 phase of cells. Includes functions associated with the growth and proliferation of cells. Some examples of these functions include colony formation, proliferation, and outgrowth of cells. Includes functions associated with cellular death and survival. Some examples of functions included in this category are cytolysis, necrosis, survival, and recovery of cells. Includes functions associated with cancer This includes any process associated with a tumour, cancer cell, or cancerous tissue, as well as any object associated with a cancer process such as transformation and metastasis. This category also includes all cancerous diseases.

Table 2 Genes differentially regulated by anti-EpCAM antibody in A2CI2, A549, and Caco-2 cells in parallel

\begin{tabular}{|c|c|c|c|c|c|c|c|}
\hline \multicolumn{3}{|c|}{ Probe set ID } & Gene symbol & Gene title & \multicolumn{3}{|c|}{ Fold change } \\
\hline |45097| at & $209304 \times$ at & $209304 \times$ at & GADD45B & Growth arrest and DNA damage-inducible gene $45 \beta$ & 18.4 & 2.5 & 2.3 \\
\hline |42293|_at & 218880_at & 218880_at & FOSL2 & FOS-like antigen 2 & 12.1 & 6.5 & 2.1 \\
\hline 1435458 at & 209193 at & 209193 at & PIMI & Pim-I oncogene & 13.9 & 4.6 & 2.1 \\
\hline 1456405 at & 227335 at & 227335 at & DIDOI & Death inducer-obliterator & -5.3 & -3.5 & -5.7 \\
\hline |454694_a_at & 237469_at & 237469_at & TOP2A & Topoisomerase $\| \alpha$ & 2.6 & 2.8 & 2.8 \\
\hline 1416958 at & 209750 at & 209750 at & NRID2 & Nuclear receptor subfamily I, group D. member 2 & 2.0 & 2.3 & 2.3 \\
\hline |455|7|_at & 218242_s_at & 222566_at & SUV42OHI & Suppressor of variegation 4-20 homologue I & -4.3 & -2.6 & -2.5 \\
\hline |424055_at & 239815_at & 225145_at & NCOA5 & Nuclear receptor coactivator 5 & -2.1 & -3.7 & -3.0 \\
\hline 1423667_at & 200769_s_at & 200769 s_at & MAT2A & Methionine adenosyltransferase $\| \alpha$ & -3.0 & -2.1 & -2.3 \\
\hline |460|79_at & 200880_at & 200880_at & DNAIAI & Dnal (Hsp40) homologue, subfamily A, member I & -2.8 & -2.0 & -2.1 \\
\hline
\end{tabular}

EpCAM = epithelial cell adhesion molecule.

transcripts from the same gene each, whereas a third definite probe set did not recognise any modulation of expression. In general, criteria defining differential expression (see Materials and Methods) are more restricted in microarray data analysis than in real-time PCR analysis, resulting in fewer genes identified as differentially expressed by microarray analysis.

\section{DISCUSSION}

Epithelial cell adhesion molecule is one of the first tumourassociated antigens identified with monoclonal antibodies (Herlyn et al, 1979). A variety of cancer therapy strategies and clinical trials have since made use of EpCAM as a target molecule for (singlechain) monoclonal and bispecific antibodies. However, only recently has it become apparent that EpCAM itself triggers intracellular signalling. Consequently, little is known so far about the possible intracellular signalling induced by EpCAM-specific antibodies. In this study, it was shown that treatment with monoclonal rat anti-mouse EpCAM antibody G8.8 induced proliferation of mouse and human lung carcinoma cells as well as human colorectal carcinoma cells. The in vitro pro-proliferative effects of anti-EpCAM treatment shown in this study are surprising, as in vivo application of anti-EpCAM antibody resulted in tumour growth suppression (Naundorf et al, 2002). However, proliferative effects have also previously been shown for the mouse anti-rat EpCAM monoclonal antibody D5.7 (Wurfel et al, 1999). Immobilised D5.7 induced proliferation in non/low-metastasising rat pancreatic adenocarcinoma, fibrosarcoma, and pheochromocytoma cell lines, which had previously been transfected with EpCAM. These data suggest that crosslinking of EpCAM by bivalent antibodies and possible subsequent di-/oligomerisation triggers pro-proliferative signals. High EpCAM expression has been associated with an increased proliferation of keratinocytes (Schon et al, 1994). On the other hand, knockdown of EpCAM by EpCAM short-interfering RNA resulted in a decrease in the cell proliferation rate in four different breast cancer cell lines (Osta et al, 2004). Thus, both treatment with EpCAM-specific antibodies 


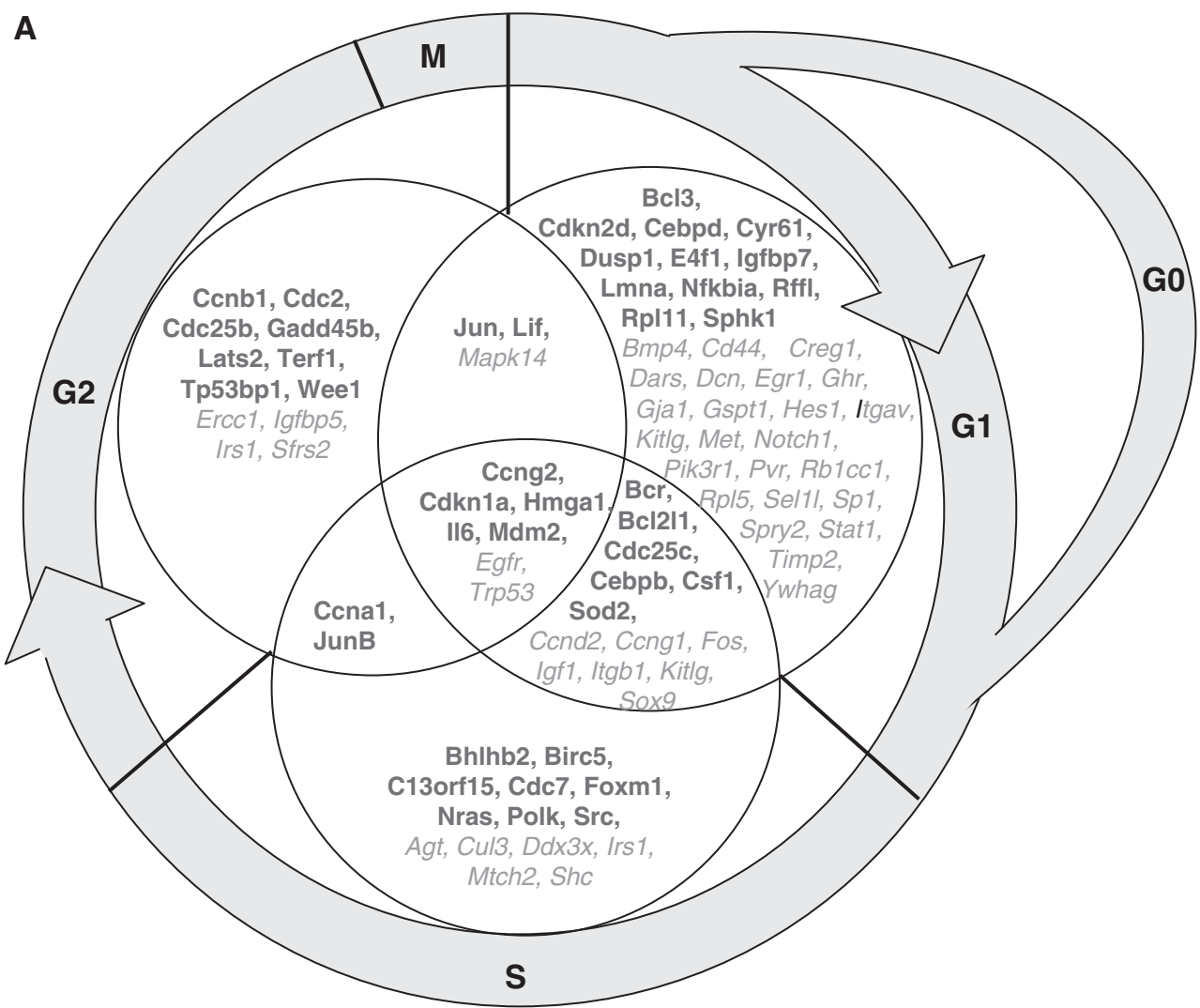

B

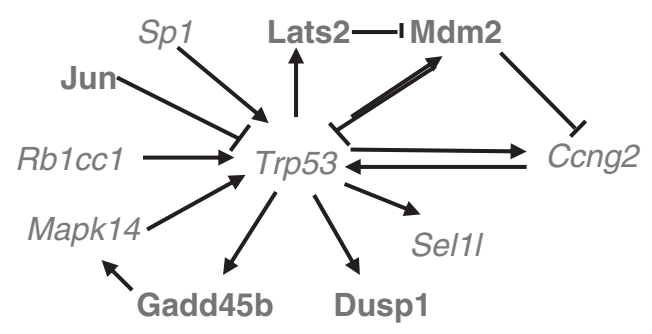

Figure 3 Cell cycle-related genes significantly regulated by anti-EpCAM treatment of $A 2 C I 2$ cells. The gene expression profile was investigated by microarray analysis, and significantly modulated genes were analysed using ingenuity software. (A) Shown are all the genes identified as functionally relevant to cell cycle regulation in the specific cell cycle phases. (B) Differentially expressed genes upon EpCAM antibody treatment of A2CI2 cells, which can be considered as p53 network. Genes induced by anti-EpCAM treatment are indicated in bold, whereas genes that were repressed are indicated in italic. EpCAM = epithelial cell adhesion molecule.

Table 3 Modulation of gene expression as detected by microarray analysis and real-time PCR

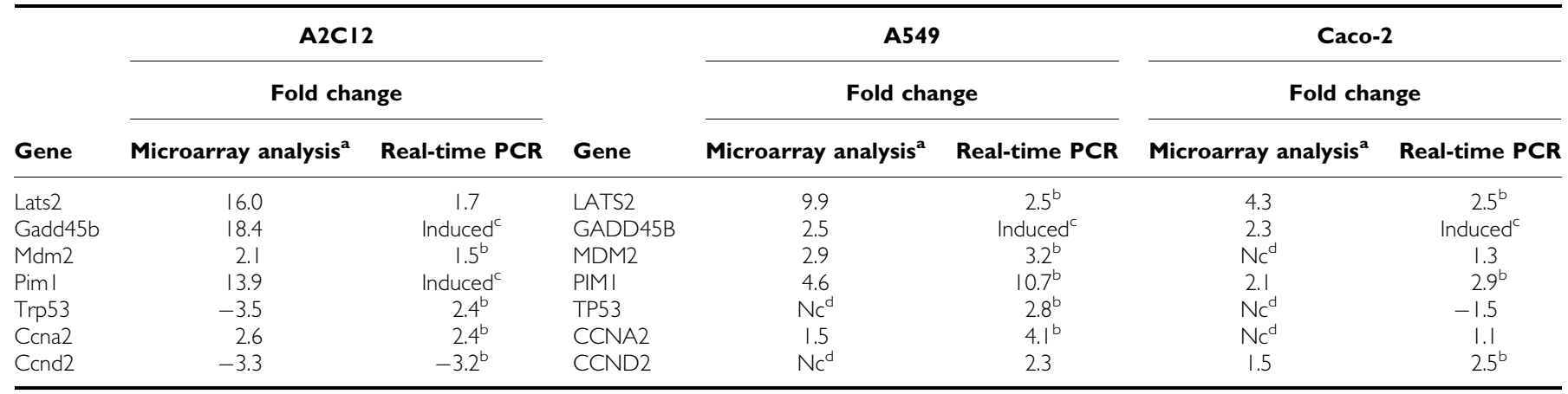

EpCAM = epithelial cell adhesion molecule. ${ }^{\text {In }}$ the case of more than one probe set identifying modulated gene expression, the highest fold change value is shown. All values $\leqslant-2$ or $\geqslant 2$ are statistically significant for $P<0.002$. Fold changes between -2 and 2 have not been considered as significant according to the applied criteria (see Materials and Methods). ${ }^{b}$ Differences of gene expression between EpCAM antibody-treated and untreated cells were statistically significant $(P<0.05)$. ${ }^{\text {CFold }}$ change could not be calculated, as no expression was detectable in the non-treated control probe. Expression of the specific PCR product in the antibody-treated probes was shown by agarose gel electrophoresis (Supplementary Figure SI). ${ }^{\mathrm{N}} \mathrm{N}$ : no change. 
and EpCAM overexpression led to an increased cell proliferation. It is tempting to speculate that spatial proximity of EpCAM molecules, either induced by crosslinking or by higher density, may be the trigger for proliferative signalling and that both processes involve similar pathways.

The exact pathways by which EpCAM modulates proliferation are, however, unknown. In this study, we performed gene expression analysis to further identify the molecules possibly involved in EpCAM antibody-induced signalling. The number of differentially expressed genes upon EpCAM treatment differed between the cell lines. The expression of most genes was found to be regulated in A2C12 and A549 cells, less in Caco-2 cells. In this study, all cell lines were treated with EpCAM-specific antibodies for the same time period. However, the investigated cell lines differ in their biological properties, for example EpCAM expression (see Figure 2) and generation time. The doubling time of the investigated cell lines ranged from $6.7 \mathrm{~h}$ for $\mathrm{A} 2 \mathrm{C} 12$ and $22 \mathrm{~h}$ for A549 to $62 \mathrm{~h}$ for Caco-2 cells. Therefore, it cannot be excluded that the quantity of differentially expressed genes depends on the biological features of the cells and adapted treatment modalities, so that for example, different antibody concentrations or different incubation times might lead to similar numbers of regulated genes.

Despite the differences in the quantity of differential gene expression, a wide overlap was detected when analysing the functions of regulated genes. Grouping the differentially regulated genes into functional processes, the cellular processes with the strongest over-representation of differentially expressed genes were the same for A1C12, A549, and Caco-2 cells. These processes include cell cycle, cell death, cellular growth and proliferation, and cancer. The identified functional groups resemble well the proliferative phenotype of cells treated with EpCAM antibody.

On the individual gene level, we concentrated on those genes that were commonly expressed in the cell lines A2C12, A549, and Caco-2. Five out of 13 commonly modulated genes were related to cell cycle-regulating or apoptotic functions. Gene expression analysis revealed that induction of proliferation was accompanied by an induction of genes whose products induce cell cycle progression (LATS2, FOSL2, and PIM1) or exert an antiapoptotic action (GADD45 and PIM1), whereas expression of pro-apoptotic genes was repressed (DIDO1). Lats2 was shown to interact physically with Mdm2, thereby inhibiting p53 ubiquitination and promoting p53 activation (Aylon et al, 2006). FOSL2 belongs to a family of transcription factors that have been implicated as regulators of cell proliferation, differentiation, and transformation (Tulchinsky, 2000). The GADD45B belongs to a group of genes that respond to environmental stresses and are involved in the regulation of growth and apoptosis. They are known to exhibit pro-apoptotic functions (Takekawa and Saito, 1998). However, recent reports showed that GADD45 genes also function in cell survival (Gupta et al, 2006). PIM1 is a serine/threonine kinase that is involved in cell cycle progression and apoptosis (Bachmann and Moroy, 2005). DIDO1 was shown to be upregulated in early apoptosis and to trigger apoptosis (Garcia-Domingo et al, 2003). These data showed that the gene expression profile well reflects the functional pro-proliferative effects of anti-EpCAM antibody.
Several differentially expressed genes can be considered as p53 network, which might play a role in the signal transduction leading to the proliferative effects of EpCAM antibody treatment. p53 expression was downregulated upon EpCAM antibody treatment possibly due to an overexpression of its regulator Mdm2. Cyclin G (Ccng2) was one of the earliest p53 target genes. Moreover, cyclin $\mathrm{G}$ directly interacts with $\mathrm{Mdm} 2$ and can stimulate the ability of PP2A to dephosphorylate Mdm2, leading to a degradation of p53 (Chen, 2002). In contrast, the p53 activator Lats2 was found to be upregulated. However, induction of other genes inhibiting p53 and repression of genes positively regulating p53 might as well contribute to the downregulation of $\mathrm{p} 53$. The transcription factor Jun is known to inhibit p53 transcription, whereas the transcription factor $\mathrm{Sp} 1$ is known to be a positive regulator of $\mathrm{p} 53$. The RB1-inducible coiled-coil 1 (Rb1cc1) was shown to stabilise p53 (Melkoumian et al, 2005). Likewise, the phosphorylation of p53 by mitogen-activated protein kinase 14 (Mapk14), also known as p38, activates p53 (Harris and Levine, 2005). Epithelial cell adhesion molecule expression has recently been associated with cell cycle regulation. Ectopic expression of EpCAM in kidney cells resulted in the upregulation of c-myc as well as cyclins A and $\mathrm{E}$ (Munz et al, 2004). In this study, the expression of cyclin A was also found to be upregulated in $\mathrm{A} 2 \mathrm{C} 12$ cells after $48 \mathrm{~h}$ of EpCAM treatment.

The application of EpCAM-specific antibodies as antineoplastic agents led to inconsistent results (reviewed in Baeuerle and Gires (2007) and Chaudry et al (2007)). The mechanisms by which anti-EpCAM antibodies exert tumour inhibition in vivo remain controversial. The cytotoxic mechanisms include antibody-dependent cell cytotoxicity mediated by natural killer cells and T lymphocytes, complement-mediated cytolysis, and opsonisation promoting phagocytosis mediated by polymorphonuclear cells. Taken together, EpCAM antibody treatment seems to make tumour cells recognisable for immune response in vivo, and the antineoplastic effects of the antibody require the immune response. These in vivo effects might overlay the possible EpCAM antibody-triggered pro-proliferative intracellular signalling seen in this study. However, future studies will have to show whether anti-EpCAM antibodies clinically applied as antitumour agents display the same pro-proliferative intracellular signalling as the antibody G8.8 used in this study.

\section{ACKNOWLEDGEMENTS}

We thank Jessica Däke for isolating the mouse lung carcinoma cell lines and Ingrid Meder and Rong Lai for their excellent technical assistance. We also thank Micromed, Munich, Germany, who generously provided us with the anti-EpCAM antibody G8.8. The financial support of Lower Saxony Ministry of Culture and Science to JB is gratefully acknowledged.

Supplementary Information accompanies the paper on British Journal of Cancer website (http://www.nature.com/bjc)

\section{REFERENCES}

Armstrong A, Eck SL (2003) EpCAM: a new therapeutic target for an old cancer antigen. Cancer Biol Ther 2: 320-326

Aylon Y, Michael D, Shmueli A, Yabuta N, Nojima H, Oren M (2006) A positive feedback loop between the p53 and Lats 2 tumor suppressors prevents tetraploidization. Genes Dev 20: $2687-2700$

Bachmann M, Moroy T (2005) The serine/threonine kinase Pim-1. Int J Biochem Cell Biol 37: 726-730

Baeuerle PA, Gires O (2007) EpCAM (CD326) finding its role in cancer. Br J Cancer 96: $417-423$
Borlak J, Meier T, Halter R, Spanel R, Spanel-Borowski K (2005) Epidermal growth factor-induced hepatocellular carcinoma: gene expression profiles in precursor lesions, early stage and solitary tumours. Oncogene 24: $1809-1819$

Chaudry MA, Sales K, Ruf P, Lindhofer H, Winslet MC (2007) EpCAM an immunotherapeutic target for gastrointestinal malignancy: current experience and future challenges. Br J Cancer 96: 1013-1019

Chen X (2002) Cyclin G: a regulator of the p53-Mdm2 network. Dev Cell 2 $518-519$ 
Fagerberg J, Hjelm AL, Ragnhammar P, Frodin JE, Wigzell H, Mellstedt H (1995) Tumor regression in monoclonal antibody-treated patients correlates with the presence of anti-idiotype-reactive $\mathrm{T}$ lymphocytes. Cancer Res 55: 1824-1827

Garcia-Domingo D, Ramirez D, Gonzalez dB, Martinez A (2003) Death inducer-obliterator 1 triggers apoptosis after nuclear translocation and caspase upregulation. Mol Cell Biol 23: 3216-3225

Gastl G, Spizzo G, Obrist P, Dunser M, Mikuz G (2000) Ep-CAM overexpression in breast cancer as a predictor of survival. Lancet 356: $1981-1982$

Guillemot JC, Naspetti M, Malergue F, Montcourrier P, Galland F, Naquet P (2001) Ep-CAM transfection in thymic epithelial cell lines triggers the formation of dynamic actin-rich protrusions involved in the organization of epithelial cell layers. Histochem Cell Biol 116: 371-378

Gupta M, Gupta SK, Hoffman B, Liebermann DA (2006) Gadd45a and Gadd45b protect hematopoietic cells from UV-induced apoptosis via distinct signaling pathways, including p38 activation and JNK inhibition. J Biol Chem 281: $17552-17558$

Hansen T, Blickwede M, Borlak J (2006) Primary rat alveolar epithelial cells for use in biotransformation and toxicity studies. Toxicol In vitro 20: $757-766$

Harris SL, Levine AJ (2005) The p53 pathway: positive and negative feedback loops. Oncogene 24: 2899-2908

Herlyn M, Steplewski Z, Herlyn D, Koprowski H (1979) Colorectal carcinoma-specific antigen: detection by means of monoclonal antibodies. Proc Natl Acad Sci USA 76: 1438-1442

Kim JW, Ye Q, Forgues M, Chen Y, Budhu A, Sime J, Hofseth LJ, Kaul R, Wang XW (2004) Cancer-associated molecular signature in the tissue samples of patients with cirrhosis. Hepatology 39: 518-527

Koprowski H, Steplewski Z, Mitchell K, Herlyn M, Herlyn D, Fuhrer P (1979) Colorectal carcinoma antigens detected by hybridoma antibodies. Somatic Cell Genet 5: 957-971

Kuhn S, Koch M, Nubel T, Ladwein M, Antolovic D, Klingbeil P, Hildebrand D, Moldenhauer G, Langbein L, Franke WW, Weitz J, Zoller M (2007) A complex of EpCAM, claudin-7, CD44 variant isoforms, and tetraspanins promotes colorectal cancer progression. Mol Cancer Res 5: $553-567$

Maaser K, Höpfner M, Jansen A, Weisinger G, Gavish M, Kozikowski AP, Weizman A, Carayon P, Riecken EO, Zeitz M, Scherübl H (2001) Specific ligands of the peripheral benzodiazepine receptor induce apoptosis and cell cycle arrest in human colorectal cancer cells. Br J Cancer 85: 1771- 1780

Melkoumian ZK, Peng X, Gan B, Wu X, Guan JL (2005) Mechanism of cell cycle regulation by FIP200 in human breast cancer cells. Cancer Res 65: $6676-6684$

Munz M, Kieu C, Mack B, Schmitt B, Zeidler R, Gires O (2004) The carcinoma-associated antigen EpCAM upregulates c-myc and induces cell proliferation. Oncogene 23: $5748-5758$

Naundorf S, Preithner S, Mayer P, Lippold S, Wolf A, Hanakam F, Fichtner I, Kufer P, Raum T, Riethmuller G, Baeuerle PA, Dreier T (2002) In vitro and in vivo activity of MT201, a fully human monoclonal antibody for pancarcinoma treatment. Int J Cancer 100: 101-110

Osta WA, Chen Y, Mikhitarian K, Mitas M, Salem M, Hannun YA, Cole DJ, Gillanders WE (2004) EpCAM is overexpressed in breast cancer and is a potential target for breast cancer gene therapy. Cancer Res 64: 5818-5824

Punt CJ, Nagy A, Douillard JY, Figer A, Skovsgaard T, Monson J, Barone C, Fountzilas G, Riess H, Moylan E, Jones D, Dethling J, Colman J, Coward L, MacGregor S (2002) Edrecolomab alone or in combination with fluorouracil and folinic acid in the adjuvant treatment of stage III colon cancer: a randomised study. Lancet 360: 671-677

Riethmuller G, Holz E, Schlimok G, Schmiegel W, Raab R, Hoffken K, Gruber R, Funke I, Pichlmaier H, Hirche H, Buggisch P, Witte J, Pichlmayr R (1998) Monoclonal antibody therapy for resected Dukes' C colorectal cancer: seven-year outcome of a multicenter randomized trial. J Clin Oncol 16: 1788 - 1794

Riethmuller G, Schneider-Gadicke E, Schlimok G, Schmiegel W, Raab R, Hoffken K, Gruber R, Pichlmaier H, Hirche H, Pichlmayr R (1994) Randomised trial of monoclonal antibody for adjuvant therapy of resected Dukes' C colorectal carcinoma. German Cancer Aid 17-1A Study Group. Lancet 343: 1177-1183
Schiechl H, Dohr G (1987) Immunohistochemical studies of the distribution of a basolateral-membrane protein in intestinal epithelial cells $(\mathrm{GZ1}-\mathrm{Ag})$ in rats using monoclonal antibodies. Histochemistry 87: $491-498$

Schon MP, Schon M, Klein CE, Blume U, Bisson S, Orfanos CE (1994) Carcinoma-associated $38-\mathrm{kD}$ membrane glycoprotein $\mathrm{MH} 99 / \mathrm{KS} 1 / 4$ is related to proliferation and age of transformed epithelial cell lines. Invest Dermatol 102: $987-991$

Seligson DB, Pantuck AJ, Liu X, Huang Y, Horvath S, Bui MH, Han KR, Correa AJ, Eeva M, Tze S, Belldegrun AS, Figlin RA (2004) Epithelial cell adhesion molecule (KSA) expression: pathobiology and its role as an independent predictor of survival in renal cell carcinoma. Clin Cancer Res 10: 2659-2669

Songun I, Litvinov SV, van de Velde CJ, Pals ST, Hermans J, van Krieken JH (2005) Loss of Ep-CAM (CO17-1A) expression predicts survival in patients with gastric cancer. $\mathrm{Br}$ J Cancer 92: $1767-1772$

Spizzo G, Obrist P, Ensinger C, Theurl I, Dunser M, Ramoni A, Gunsilius E, Eibl G, Mikuz G, Gastl G (2002) Prognostic significance of Ep-CAM AND Her-2/neu overexpression in invasive breast cancer. Int J Cancer 98: $883-888$

Spizzo G, Went P, Dirnhofer S, Obrist P, Moch H, Baeuerle PA, MuellerHolzner E, Marth C, Gastl G, Zeimet AG (2006) Overexpression of epithelial cell adhesion molecule (Ep-CAM) is an independent prognostic marker for reduced survival of patients with epithelial ovarian cancer. Gynecol Oncol 103: 483-488

Spizzo G, Went P, Dirnhofer S, Obrist P, Simon R, Spichtin H, Maurer R, Metzger U, von Castelberg B, Bart R, Stopatschinskaya S, Kochli OR, Haas P, Mross F, Zuber M, Dietrich H, Bischoff S, Mirlacher M, Sauter G, Gastl G (2004) High Ep-CAM expression is associated with poor prognosis in node-positive breast cancer. Breast Cancer Res Treat 86: $207-213$

Stoecklein NH, Siegmund A, Scheunemann P, Luebke AM, Erbersdobler A, Verde PE, Eisenberger CF, Peiper M, Rehders A, Esch JS, Knoefel WT, Hosch SB (2006) Ep-CAM expression in squamous cell carcinoma of the esophagus: a potential therapeutic target and prognostic marker. $B M C$ Cancer 6: 165

Takekawa M, Saito H (1998) A family of stress-inducible GADD45-like proteins mediate activation of the stress-responsive MTK1/MEKK4 MAPKKK. Cell 95: $521-530$

Tulchinsky E (2000) Fos family members: regulation, structure and role in oncogenic transformation. Histol Histopathol 15: 921-928

Varga M, Obrist P, Schneeberger S, Muhlmann G, Felgel-Farnholz C, Fong D, Zitt M, Brunhuber T, Schafer G, Gastl G, Spizzo G (2004) Overexpression of epithelial cell adhesion molecule antigen in gallbladder carcinoma is an independent marker for poor survival. Clin Cancer Res 10: $3131-3136$

Went P, Vasei M, Bubendorf L, Terracciano L, Tornillo L, Riede U, Kononen J, Simon R, Sauter G, Baeuerle PA (2006) Frequent high-level expression of the immunotherapeutic target Ep-CAM in colon, stomach, prostate and lung cancers. Br J Cancer 94: 128-135

Winter MJ, Cirulli V, Briaire-de Bruijn IH, Litvinov SV (2007) Cadherins are regulated by Ep-CAM via phosphaditylinositol-3 kinase. Mol Cell Biochem 302: 19-26

Winter MJ, Nagelkerken B, Mertens AE, Rees-Bakker HA, Briaire-de Bruijn IH, Litvinov SV (2003a) Expression of Ep-CAM shifts the state of cadherin-mediated adhesions from strong to weak. Exp Cell Res 285: $50-58$

Winter MJ, Nagtegaal ID, van Krieken JH, Litvinov SV (2003b) The epithelial cell adhesion molecule (Ep-CAM) as a morphoregulatory molecule is a tool in surgical pathology. Am J Pathol 163: 2139-2148

Wurfel J, Rosel M, Seiter S, Claas C, Herlevsen M, Weth R, Zoller M (1999) Metastasis-association of the rat ortholog of the human epithelial glycoprotein antigen EGP314. Oncogene 18: 2323-2334

Yamashita A, Hakura A, Inoue H (1999) Suppression of anchorageindependent growth of human cancer cell lines by the drs gene. Oncogene 18: $4777-4787$

Yamashita T, Budhu A, Forgues M, Wang XW (2007) Activation of hepatic stem cell marker EpCAM by Wnt-beta-catenin signaling in hepatocellular carcinoma. Cancer Res 67: 10831-10839 